\title{
Analysis of Stability in Enzyme Biosensor Based on Michaelis-Menten Model with Time Delays
}

\author{
A. KLOS-WitKowska ${ }^{a, *}$, V. MARTSEnYUK ${ }^{a}$ And V. KARPINSKYI ${ }^{b}$ \\ ${ }^{a}$ Faculty of Mechanical Engineering and Computer Science, Department of Computer Science and Automatics, \\ University of Bielsko-Biała, Willowa 2, 43-309 Bielsko-Biała, Poland \\ ${ }^{b} 3 \mathrm{D}$ Scanners UK Ltd, the Techno Centre, Coventry, United Kingdom
}

(Received February 5, 2018)

\begin{abstract}
This work offers modelling of enzyme biosensors based on systems of partial differential equations with time delays, including the Michaelis-Menten dynamics for chemical reactions. We offer the numerical approach for stability investigation of steady states of such a model by solving corresponding boundary value problems. The corresponding software is developed using $\mathrm{R}$ package.
\end{abstract}

DOI: 10.12693/APhysPolA.135.375

PACS/topics: enzyme biosensor, partial differential equations, Michaelis-Menten dynamics, time delays, stability

\section{Introduction}

Intensive development of industry, use of chemicals in agriculture, and the growth in the world population have an increasingly negative impact on the environment. As a result, increased risk of diseases in humans and animals have been noticed. Therefore, it is necessary to systematically control the current state of the environment, as well as human health. Traditional used methods of detection are: high performance liquid chromatography (HPLC) which is coupled with fluorometric UV detection, calorimetric immunoassay, and liquid chromatography-mass spectroscopy (LC-MS) — which are not perfect because they require uses of expensive laboratory equipments, highly trained personnels, and usually take long time.

In this modern era, when the quality of the measurement is one of the major concern, biosensors are an interesting alternative to the commonly used long-term detection methods. These sensors are easy to use, and their applications do not require specialized laboratory or trained personnels. For these reasons, they are convenient to existing needs. Biosensors are commonly used for environmental monitoring - to study water pollution [1], to detect toxic substances in the defence industry [2], to detect the presence of allergic components in the food industry [3], and also for diagnosis in medical fields [4]. Its universal acceptance is an area worthy of attention from a financial perspective. On the basis of the economic analysis carried out by Transparency Market Research, it is estimated that the value of the biosensor market in 2018 will reach 18.9 trillion USD [5]. The current trends in the development of biosensors points toward several possible research directions: use of the achievements of nanotechnology for improvement of biosensor

*corresponding author; e-mail: awitkowska@ath.bielsko.pl construction [6], miniaturization, which is currently used in biosensors [7], use of graphene [8], and finally, study on improvement of sensitivity, selectivity, reproducibility, and reliability of existing biosensors [9]. Among these, study on biosensor stability could be highlighted, specially on receptor layer stability, as the biological components are unstable in time. According to the definition of International Union of Pure and Applied Chemistry (IUPAC), a biosensor is: a self-contained integrated device capable of proving specific quantitative analytical information using elements retained in direct spatial contact with transduction elements [10]. Biosensor structure is based on the receptor layer and transducer layer. The receptor layer is a sensitive, selective matrix, usually containing a biological element. On its surface (according to the lock-fit mechanism), there is an uptake of a substance called an analyte. Those component might be: antibodies, antigens, nucleic acids, proteins, bacteria, and lastly, enzymes.

\section{Stability in enzyme based biosensor}

Stability of receptor layer components are very important, because it determines the time of biosensor utility. In the context of biosensors, two types of stability can be distinguished: self stability and operational stability. Self stability is defined as the enhancement or improvement of activity retention of an enzyme, protein, diagnosis or a device, when stored under specific conditions [11]. Operational stability is the retention of activity when in use [11]. Stability of a sensitive element located in the receptor layer of the sensor, and stability related to the activity of biosensor matrix components during biosensor use, determine the usable life of the device. With the passage of time, the activity of the ingredients decrease. For example, conformational changes in proteins results in the change of enzymes activity. Therefore, in recent years, the research work regarding the improvement of the biosensor stability have 
been observed [12]. Among them, special attention is directed to the enzymatic biosensors [13], where, with the use of special immobilization techniques, enzymes are put into bio-receptor layer [14]:

Adsorption: This method is cheap and easy. One of the advantage is possibility of subsequent enzyme regeneration. However, the disadvantage is poor operational and storage stability. With the help of this method, simple deposition of enzyme onto the electrode materials and attachment results in weak bonds, as Van der Waals electrostatic interaction takes place [14-16].

Entrapment: This method increases operational and storage stability. However, the enzyme's activity is preserved during the immobilization process. Photo-polymerizable matrix, electro-polymerizable films, and sol-gel matrices are utilized in the entrapment processes [17-19].

Covalent immobilization: This method improves uniformity, density and distribution of bio-elements as well as reproducibility and homogeneity of the surface. In enzyme based biosensors, the functional groups in the enzyme (nucleophilic functional groups present in amino acid side chains of protein, such as, amino, carboxylic, imidazole, thiol, hydro etc.) are used for coupling.

Affinity interaction: This method is based on affinity interaction between functional groups (lectin, metal chelate), on achieved electrode surface and affinity tag (carbohydrate residues, biotin, histidine cysteine). Adsorption can involve stronger bonds even if they are not as strong as the covalent ones [15].

Cross-linking: This method is attractive due to its simplicity and the strong chemical bonding [15]. Most common cross-linking in biosensor application is glutaldehyde which couples with lysine amino groups of enzymes [20]. Cross-linkings or covalent bonds in the enzymes support material surfaces. However, enzymes become less mobile and dramatically decrease their activities [15].

\section{Biosensor model with time delays}

According to the approach offered in the paper [21], a biosensor can be considered as an enzyme electrode, containing a membrane with immobilized enzymes applied onto the surface of the electrochemical transducer. We assume a symmetrical geometry of the electrode, and homogeneous distribution of immobilized enzymes in the enzyme membrane.

As a prototype we used so called CEC model as discussed in [21]. According to this model, the substrate $(S)$ is enzymatically $\left(E_{1}\right)$ converted to the product $\left(P_{1}\right)$, followed by the electrochemical conversion of the product $P_{1}$ to another product $\left(P_{2}\right)$ that, in turn, is again enzymatically $\left(E_{2}\right)$ converted back to $P_{1}$ :

$$
\begin{aligned}
& S \stackrel{E_{1}}{\longrightarrow} P_{1}, \\
& P_{1} \longrightarrow P_{2}, \\
& P_{2} \stackrel{E_{2}}{\longrightarrow} P_{1} .
\end{aligned}
$$

Now, enzyme-catalysed reactions (1), (1) and electrochemical reaction (2) are performed with some delays in time $\tau_{1}, \tau_{2}>0[22,23]$ :

$$
\begin{aligned}
& S \stackrel{E_{1} \text {, with delay } \tau_{0}}{\longrightarrow} P_{1}, \\
& P_{1} \stackrel{\text { with delay } \tau_{1}}{\longrightarrow} P_{2}, \\
& P_{2} \stackrel{E_{2} \text {, with delay } \tau_{2}}{\longrightarrow} P_{1} .
\end{aligned}
$$

Now, coupling the enzyme-catalysed reactions (1), (3) and electrochemical reaction (2), with the one dimensional in-space diffusion, described by Fick's law, and the use of Michelis-Menten model to describe kinetic behaviour of enzyme reaction, lead to the following equations $(t>0,0<x<d)$ :

$$
\begin{aligned}
& \frac{\partial S}{\partial t}=D_{s} \frac{\partial^{2} S}{\partial x^{2}}-\frac{V_{0} S\left(x, t-\tau_{0}\right)}{K_{0}+S\left(x, t-\tau_{0}\right)}, \\
& \frac{\partial P_{1}}{\partial t}=D_{P_{1}} \frac{\partial^{2} P_{1}}{\partial x^{2}}+\frac{V_{0} S\left(x, t-\tau_{0}\right)}{K_{0}+S\left(x, t-\tau_{0}\right)} \\
& -\frac{V_{1} P_{1}\left(x, t-\tau_{1}\right)}{K_{1}+P_{1}\left(x, t-\tau_{1}\right)}+\frac{V_{2} P_{2}\left(x, t-\tau_{2}\right)}{K_{2}+P_{2}\left(x, t-\tau_{2}\right)} \\
& \frac{\partial P_{2}}{\partial t}=D_{P_{2}} \frac{\partial^{2} P_{2}}{\partial x^{2}}+\frac{V_{1} P_{1}\left(x, t-\tau_{1}\right)}{K_{1}+P_{1}\left(x, t-\tau_{1}\right)} \\
& -\frac{V_{2} P_{2}\left(x, t-\tau_{2}\right)}{K_{2}+P_{2}\left(x, t-\tau_{2}\right)},
\end{aligned}
$$

where $x$ and $t$ stand for space and time, respectively. $S(x, t)$ and $P_{i}(x, t)$ denote the concentration functions of the substrate $S$ and product $P_{i}$, respectively. $V_{i}$ is the maximal substrate (enzymatic) rate, $K_{i}$ is the Michaelis constant, $d$ is the thickness of the enzyme membrane, $D_{s}$ and $D_{P_{i}}$ are the diffusion coefficients, with $i=0,1,2$.

Let $x=0$ represent the electrode surface and $x=d$, the bulk solution/membrane interface. The operation of the biosensor starts when some substrate appears over the surface of the enzyme membrane. This is used with the initial conditions

$$
\begin{aligned}
& \left(t \in\left[-\tau_{M}, 0\right], \tau_{M}=\max \left(\tau_{0}, \tau_{1}, \tau_{2}\right)\right): \\
& S(x, t)=0, \quad 0 \leq x<d, \quad S(d, t)=S_{0}, \\
& P_{i}(x, t)=0, \quad 0 \leq x<d, \quad i=1,2,
\end{aligned}
$$

where $S_{0}$ is the concentration of substrate in the bulk solution.

The electrode potential is chosen to keep the zero concentration of the product $P_{1}$ at the electrode surface. The rate of the product $P_{2}$ generation at the electrode is proportional to the rate of conversion of the product $P_{1}$. When the substrate is well-stirred outside the membrane, the diffusion layer remains at a constant thickness $(0<x<d)$. Consequently, the concentration of the substrate as well as both products over the enzyme surface (bulk solution/membrane interface) remain 
constant, while the biosensor contacts the solution of substrate. This is used in the boundary conditions $(t>0)$, given by,

$$
\begin{aligned}
& \left.\frac{\partial S}{\partial x}\right|_{x=0}=0, \\
& S(d, t)=S_{0}, \\
& \left.D_{P_{2}} \frac{\partial P_{2}}{\partial x}\right|_{x=0}=-\left.D_{P_{1}} \frac{\partial P_{1}}{\partial x}\right|_{x=0}, \\
& P_{i}(d, t)=0, \quad i=1,2 \\
& P_{1}(0, t)=0 .
\end{aligned}
$$

The biosensor current depends upon the flux of the product $P_{1}$ at the electrode surface, i.e. at the interface, $x=0$. Consequently, the density $i_{\mathrm{CEC}}(t)$ of the current at time $t$ can be obtained explicitly from Faraday's and Fick's laws using the flux of the concentration of the product $P_{1}$ at the surface of the electrode,

$$
\begin{aligned}
& i_{\mathrm{CEC}}(t)=\left.n_{e} F D_{P_{2}} \frac{\partial P_{2}}{\partial x}\right|_{x=0}= \\
& -\left.n_{e} F D_{P_{1}} \frac{\partial P_{1}}{\partial x}\right|_{x=0},
\end{aligned}
$$

where $n_{e}$ is the number of electrons involved in a charge transfer at the electrode surface, and $F$ is the Faraday constant, $F=96485 \mathrm{C} / \mathrm{mol}$. We assume that systems (7)-(17) approaches a steady-state as $t \rightarrow \infty$ :

$$
\lim _{t \rightarrow \infty} i_{\mathrm{CEC}}(t)=I_{\mathrm{CEC}},
$$

where $I_{\mathrm{CEC}}$ is the steady-state biosensor current.

\section{Analysis of biosensor stability based on Michaelis-Menten model}

Let us consider biosensor model at the electrode surface, i.e. $x=0$. In such a case, by taking into account Eqs. (10)-(18), systems (7)-(9) are transformed into a system of differential equations with delays

$$
\begin{aligned}
\frac{\mathrm{d} S}{\mathrm{~d} t} & =-\frac{V_{1} S\left(0, t-\tau_{1}\right)}{K_{1}+S\left(0, t-\tau_{1}\right)}, \\
\frac{\mathrm{d} P_{1}}{\mathrm{~d} t} & =\frac{V_{0} S\left(0, t-\tau_{0}\right)}{K_{0}+S\left(0, t-\tau_{0}\right)}+\frac{V_{2} P_{2}\left(0, t-\tau_{2}\right)}{K_{2}+P_{2}\left(0, t-\tau_{2}\right)}, \\
\frac{\mathrm{d} P_{2}}{\mathrm{~d} t} & =-\frac{V_{2} P_{2}\left(0, t-\tau_{2}\right)}{K_{2}+P_{2}\left(0, t-\tau_{2}\right)} .
\end{aligned}
$$

Hence, we get only one trivial steady state, $\left(S^{0}, P_{1}^{0}, P_{2}^{0}\right)=(0,0,0)$ at $t \rightarrow \infty$.

Let us now consider biosensor model at the membrane interface, i.e. $x=d$. In this case, by taking into account Eqs. (10)-(18), systems (7)-(9) are transformed into the system of differential equations with delays

$$
\frac{\mathrm{d} S}{\mathrm{~d} t}=-\frac{V_{1} S\left(d, t-\tau_{1}\right)}{K_{1}+S\left(d, t-\tau_{1}\right)},
$$

$$
\begin{aligned}
& \frac{\mathrm{d} P_{1}}{\mathrm{~d} t}=\frac{V_{0} S\left(d, t-\tau_{0}\right)}{K_{0}+S\left(d, t-\tau_{0}\right)}-\frac{V_{1} P_{1}\left(d, t-\tau_{1}\right)}{K_{1}+P_{1}\left(d, t-\tau_{1}\right)} \\
& +\frac{V_{2} P_{2}\left(d, t-\tau_{2}\right)}{K_{2}+P_{2}\left(d, t-\tau_{2}\right)}, \\
& \frac{\mathrm{d} P_{2}}{\mathrm{~d} t}=-\frac{V_{2} P_{2}\left(d, t-\tau_{2}\right)}{K_{2}+P_{2}\left(d, t-\tau_{2}\right)} .
\end{aligned}
$$

Hence, we again get only trivial steady state, $\left(S^{d}, P_{1}^{d}, P_{2}^{d}\right)=(0,0,0)$ at $t \rightarrow \infty$.

While analysing (7)-(9) at arbitrary $0<x<d$, we get steady states, $\left(S, P_{1}, P_{2}\right)=\left(0, P_{1}, P_{2}\right)$, where

$$
\frac{V_{1} P_{1}}{K_{1}+P_{1}}=\frac{V_{2} P_{2}}{K_{2}+P_{2}} \text {. }
$$
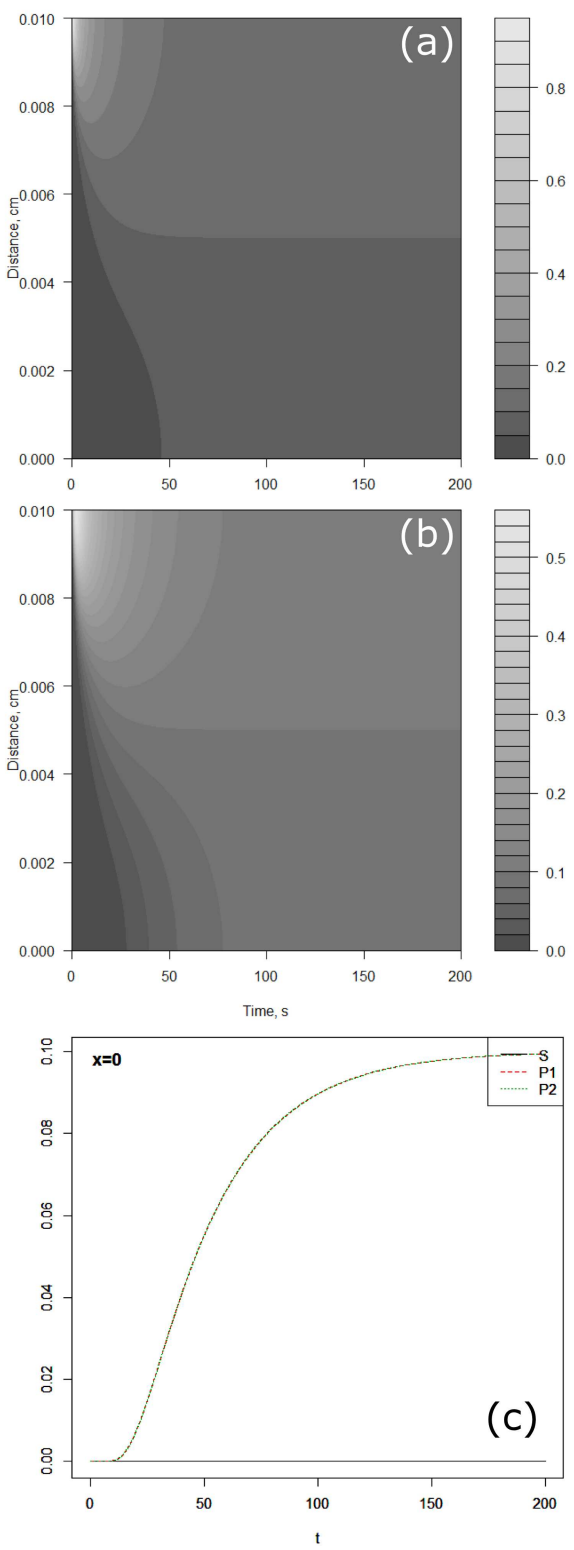

Fig. 1. Biosensor model solution at $D_{S}=D_{P_{1}}=$ $D_{P_{2}}=3.0 \times 10^{-7} \mathrm{~cm}^{2} / \mathrm{s}$ : (a) concentration of $P_{1}$, (b) concentration of $P_{2}$, (c) concentrations of $S, P_{1}$ and $P_{2}$ at $x=0$ 


\section{Numerical work}

Numerical simulations were carried out using $R$ package software and the library deSolve. For the numerical simulations, we have chosen the following values of parameters of the model (7)-(9): thickness, $d=0.01 \mathrm{~cm}$; $V_{0}=V_{1}=V_{2}=100 \mathrm{nmol} /\left(\mathrm{cm}^{3} \mathrm{~s}\right) ; \tau_{0}=\tau_{1}=\tau_{2}=0.1 \mathrm{~s} ;$ Michaeli's constants, $K_{0}=K_{1}=K_{2}=100 \mathrm{nmol} / \mathrm{cm}^{3}$; $S_{0}=20 \mathrm{nmol} / \mathrm{cm}^{3}$.

The diffusion parameters appeared to be the most important factors while investigating the model stability. For example, by taking $D_{S}=D_{P_{1}}=D_{P_{2}}=$ $3.0 \times 10^{-7} \mathrm{~cm}^{2} / \mathrm{s}$, we get stable steady state in the form (19) (Fig. 1).

Setting $D_{S}=D_{P_{1}}=D_{P_{2}}=3.0 \times 10^{-8} \mathrm{~cm}^{2} / \mathrm{s}$ results in the steady state of the system becoming unstable (Fig. 2).
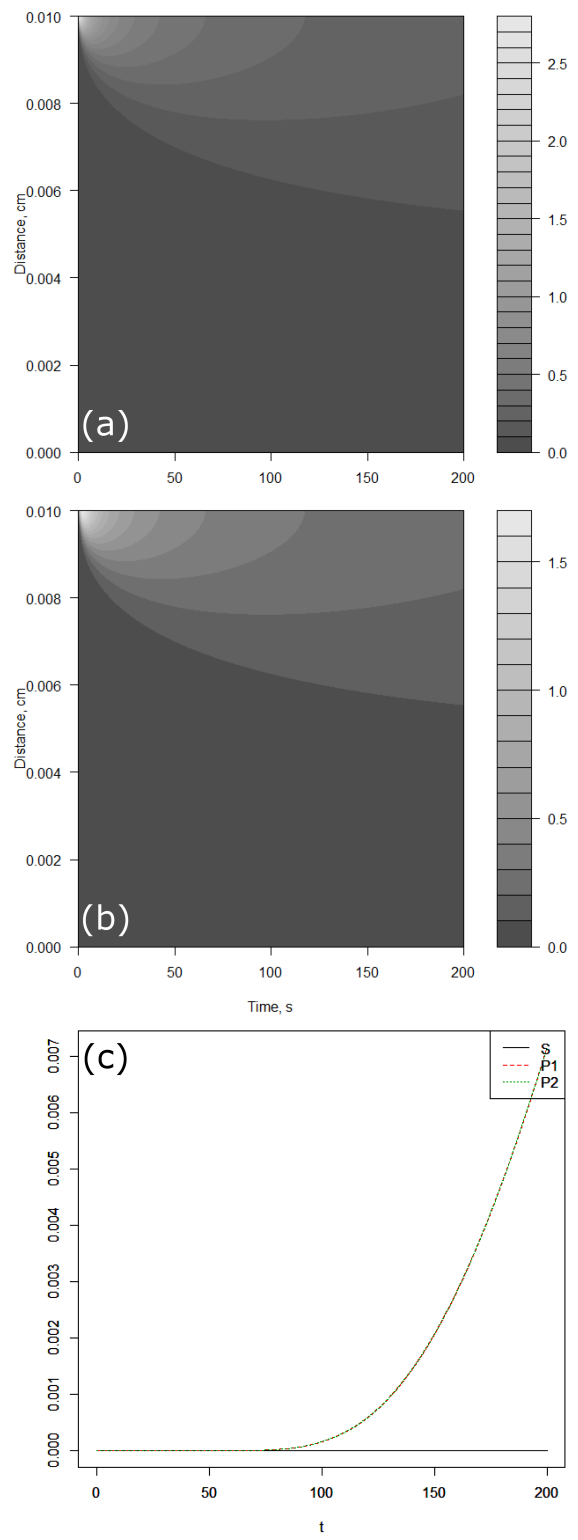

Fig. 2. Biosensor model solution at $D_{S}=D_{P_{1}}=$ $D_{P_{2}}=3.0 \times 10^{-8} \mathrm{~cm}^{2} / \mathrm{s}$ : (a) concentration of $P_{1}$, (b) concentration of $P_{2},(\mathrm{c})$ concentrations of $S, P_{1}$ and $P_{2}$ at $x=0$.

\section{Conclusions}

Thus in this paper, we have offered a spatial-temporal model of biosensor devices considering time delays. There have been a lot of attempts to develop a models of enzyme biosensors based on partial differential equations. But the idea of delays in time is novel for the application of those models. As it has been shown in previous works, primarily, for the problems of pharmacokinetics, by considering time delays, we can observe new phenomena in qualitative behaviour of such systems. One of the most important thing in development of biosensors is the stability characteristics. The models with delays are so-called infinite-dimensional systems and they have more complex behaviour as compared to finite-dimensional ones.

Thus, models considered in this paper allow us to investigate biosensor models from the viewpoint of hereditary systems, i.e., to consider the dependence of the future states of biosensor by acknowledging not only current states, but also past ones. For future work, we focus on the development of stability criteria in biosensor models based on systems with delays.

\section{References}

[1] P. Gautam, S. Prachi, K. Amrita, D. Madathil, B. Nair, Int. J. Environm. Sci. 2, 1565 (2012).

[2] J.J. Gooding, Anal. Chim. Acta 559, 137 (2006).

[3] P. Poltronieri, V. Mezzolla, E. Primiceri, G. Maruccio, Foods 3, 511 (2014).

[4] A. Kłos-Witkowska, Acta Biochim. Pol. 63, 215 (2016).

[5] Transparency Market Research, Biosensors Market (Electrochemical, Optical, Piezoelectric \& Thermistor) - Global Industry Analysis, Size, Share, Growth, Trends and Forecast, 2012-2018, available on 2013-06-27.

[6] X. Zang, Q. Guo, D. Cui, Sensors (Basel) 9, 1033 (2009).

[7] B. Derkus, Biosens. Bioelectron. 79, 901 (2016).

[8] C.S. Park, H. Yoon, O.S. Kwon, J. Industr. Eng. Chem. 38, 13 (2016).

[9] A. Hasan, M. Nurunnabi, M. Morshed, A. Paul, A. Polini, T. Kuila, M. Al Hariri, Y.-K. Lee, A.A. Jaffa, BioMed Res. Int. 2014, 307519 (2014).

[10] D. Thevenot, K. Toht, R. Dust, G. Wilson, Pure Appl. Chem. 71, 2333 (1999).

[11] T. Gibson, Analysis 27, 630 (1999).

[12] T. Itoh, T. Shimomura, A. Hayashi, A. Yamaguchi, N. Teramae, M. Ono, T. Tsunoda, F. Mizukami, G.D. Stucky, T.A. Hanaoka, Analyst 139, 4654 (2014).

[13] J. Njagi, S. Andreescu, Biosens. Bioelectron. 23, 168 (2007).

[14] A. Kłos-Witkowska, Pol. J. Environm. Stud. 24, 19 (2015).

[15] B. Leca-Bouvier, L. Blum, in: Recognition Receptors in Biosensors, Ed. Zourob, Springer Science + Business Media LLC 2010. 
[16] W. Putzbach, N. Ronkainen, Sensors 13, 4811 (2013).

[17] N. Starodub, A. Rebviev, Bioelectrochemistry 71, 29 (2007).

[18] S. Psoma, P. Wal, N. Rooij, Proced. Eng. 25, 1369 (2011).

[19] R. Gupta, N. Chaudhury, Biosens. Bioelectron. 22, 2387 (2007).

[20] R. Monosik, M. Stredansky, E. Sturdik, Acta Chem. Slov. 5, 109 (2012).
[21] R. Baronas, J. Kulys, F. Ivanauskas, Sensors 4, 20 (2004).

[22] V. Martsenyuk, I. Andrushchak, J. Automat. Inform. Sci. 41, 24 (2009).

[23] V. Martsenyuk, I. Andrushchak, I. Melenchuk, J. Automat. Inform. Sci. 44, 32 (2012). 